\title{
Millian superiorities and the repugnant conclusion
}

Jensen, Karsten Klint

Published in:

Utilitas

DOI:

10.1017/S0953820808003154

Publication date:

2008

Document version

Publisher's PDF, also known as Version of record

Citation for published version (APA):

Jensen, K. K. (2008). Millian superiorities and the repugnant conclusion. Utilitas, 20(3), 279-300.

https://doi.org/10.1017/S0953820808003154 


\title{
Millian Superiorities and the Repugnant Conclusion
}

\author{
KARSTEN KLINT JENSEN \\ University of Copenhagen, Denmark
}

\begin{abstract}
James Griffin has considered a form of superiority in value that is weaker than lexical priority as a possible remedy to the Repugnant Conclusion. In this article, I demonstrate that, in a context where value is additive, this weaker form collapses into the stronger form of superiority. And in a context where value is non-additive, weak superiority does not amount to a radical value difference at all. These results are applied on one of Larry Temkin's cases against transitivity. I demonstrate that Temkin appeals to two conflicting notions of aggregation. I then spell out the consequences of these results for different interpretations of Griffin's suggestion regarding population ethics. None of them comes out very successful, but perhaps they nevertheless retain some interest.
\end{abstract}

\section{INTRODUCTION}

John Stuart Mill famously introduced the notion of superiority of quality of a pleasure, claiming that ${ }^{1}$

[i]f one of the two [pleasures] is, by those who are competently acquainted with both, placed so far above the other that they prefer it, even though knowing it to be attended with a greater amount of discontent, and would not resign it for any quantity of the other pleasure which their nature is capable of, we are justified in ascribing to the preferred enjoyment a superiority in quality, so far outweighing quantity as to render it, in comparison, of small account.

In recent decades, such superiority relations between different objects of value have been the subject of interest, probably because James Griffin, Derek Parfit and others have considered them a possible remedy to the Repugnant Conclusion in population ethics.

Parfit states different versions of the Repugnant Conclusion, ${ }^{2}$ but what they seem to have in common is this: suppose we have a scale of welfare. Consider some number of people $n$ all living on a very high level $\boldsymbol{a}$. For any positive level of welfare, $\boldsymbol{z}$, however low, a population of $m$ people at $\boldsymbol{z}$ is better than $n$ people at $\boldsymbol{a}$, provided $m$ is large enough. Parfit and many others consider this conclusion repugnant. It follows straightforwardly from the Utilitarian Total Principle (if $m \boldsymbol{z}>n \boldsymbol{a}$ and hence if $m>n \boldsymbol{a} / \boldsymbol{z})$. But according to Parfit, it follows from

\footnotetext{
1 John Stuart Mill, Utilitarianism, 1861, quoted from Utilitarianism, On Liberty, Considerations on Representative Government (London, 1993), p. 9.

${ }^{2}$ Derek Parfit, Reasons and Persons (Oxford, 1984), pp. 338, 419-41.
}

(C) 2008 Cambridge University Press

doi:10.1017/S0953820808003154

CAMBRIDGE
Utilitas Vol. 20, No. 3, September 2008

Printed in the United Kingdom 
any reasonable principle of beneficence, ${ }^{3}$ provided that mere addition of people at a positive level of welfare does not make an outcome worse and that the principle if $\boldsymbol{y}$ is not worse than $\boldsymbol{x}$ and $\boldsymbol{z}$ is better then $\boldsymbol{y}$, then $\boldsymbol{z}$ is better than $\boldsymbol{x}^{\prime}$ holds for betterness.

In a note, Griffin writes: ${ }^{4}$

[...] That our reasoning carries us to New $\mathrm{Z}$ is The Repugnant Conclusion.

But does it? [...] there is another possibility confined entirely to the reasoning about beneficence. Parfit's argument seems implicitly to employ a tottingup conception of measuring well-being; it treats well-being as measurable on a single continuous additive scale, where low numbers, if added to themselves often enough, must become larger than any initial, larger number. But this seems not true in prudential cases, and it would seem likely that this incommensurability in prudential values would get transferred to interpersonal calculation. Perhaps it is better to have a certain number of people at a certain high level than a very much larger number at a level where life is just worth living. Then we might wish to stop the slide [...] at that point along the line where people's capacity to appreciate beauty, to form deep loving relationships, to accomplish something with their lives beyond just staying alive... all disappear.

Griffin points to an implicit assumption as to the measurement of welfare. He claims that Parfit's arguments to the effect that we cannot avoid the Repugnant Conclusion implicitly assume welfare to be measurable on a continuous additive scale satisfying what is known as the Archimedean property of real numbers: for any positive number $x$, no matter how small, and for any number $y$, no matter how large, there exists an integer $n$, such that $n x \geq y$. This simply means that any two (positive) levels of welfare are commensurable, i.e. their ratio is not infinite.

But reflection on the measurement of welfare suggests that this assumption is not fulfilled. Presumably, then, a certain low level $\boldsymbol{z}$ could be infinitely small compared with other, higher levels, for instance $\boldsymbol{a}$. And it would seem to follow that this level could never add up to the high level, that is, the total $n \boldsymbol{a}$ would necessarily be greater than the total $m \boldsymbol{z}$, no matter how large $m$ is. Therefore, the Utilitarian Total Principle does not imply the Repugnant Conclusion. At least this is how I shall understand Griffin's suggestion.

Somewhat strangely, no one appears to have taken this suggestion seriously. Roger Crisp is an exception. ${ }^{5}$ He explicitly draws out the

\footnotetext{
${ }^{3}$ A 'reasonable' principle of distribution, in this context, is a principle which implies that if one of two outcomes with the same people has a greater total of welfare and it has welfare more equally distributed, then it is better.

${ }^{4}$ James Griffin, Well-Being: Its Meaning, Measurement and Moral Importance (Oxford, 1986), p. 340 (n. 27).

${ }^{5}$ Roger Crisp, 'Ideal Utilitarianism: Theory and Practice' (DPhil. Thesis, Oxford University, 1988), pp. 177-8.
} 
consequence that some form of discontinuity will block that the Repugnant Conclusion follows from Total Utilitarianism. However, he also identifies this position as a version of Parfit's Lexical View. ${ }^{6}$ This seems to me a confusion of two clearly distinct positions. As I understand the Lexical View, there is a standard (Archimedean) scale of welfare; but on this scale we then determine two levels, such that lives above the higher level are assigned a weight which lexically dominates the weight of lives below the lower level.

The trouble with this view is that it requires some justification to claim that the welfare of some persons should weigh differently from that of others. It can hardly be said to be a concern for beneficence to assign less weight to low levels. And the weights have nothing to do with considerations of equality: in the outcomes in question, there is complete equality. It is precisely because it avoids this problem that Griffin's suggestion deserves attention.

Griffin introduces two superiority relations that could account for welfare not being measurable on a scale fulfilling the Archimedean property. One is trumping: 'any amount of $A$ outranks any amount of $B$ ' and the other is the weaker discontinuity: 'enough of $A$ outranks any amount of $B$ '. ${ }^{7} \mathrm{He}$ considers the latter more plausible and therefore his argument is based on discontinuity.

In this article, I shall present some general results on the properties of this value superiority relation between objects. As we shall see, it behaves very differently, depending on whether value is additive or not. In an additive context, discontinuity collapses into trumping. And in a non-additive context, discontinuity - perhaps counter intuitively does not imply a radical value difference at all. I illustrate these results on a case of Larry Temkin's against transitivity. I then go on to spell out the consequences of these results for different interpretations of Griffin's suggestion regarding population ethics.

\section{SUPERIORITY IN VALUE WHEN VALUE IS ADDITIVE}

This section and the next build on and complement a paper by Arrhenius and Rabinowicz. ${ }^{8}$ The framework is this: suppose there is a countable set of objects. I assume that there is a concatenation procedure by which it is possible to form a new object by conjoining a finite number of separate objects into one whole. This includes the possibility of conjoining an object $\boldsymbol{e}$ a finite number of times $m$ with

${ }_{6}$ Parfit, Reasons and Persons, p. 188.

7 Griffin, Well-Being, pp. 83-6.

8 Gustaf Arrhenius and Wlodek Rabinowicz, 'Millian Superiorities', Utilitas 17 (2005), pp. 127-46. 
an object exactly like itself; such an object is designated by ' $m \boldsymbol{e}$ '. The domain is closed under concatenation. ${ }^{9}$

On the domain of objects, there is a weak betterness relation, - is at least as good as -. Strict betterness and equivalence relations are defined in the usual way. ${ }^{10}$ This weak betterness relation is assumed to be transitive ${ }^{11}$ and complete. ${ }^{12}$ It is further assumed that concatenation is value-increasing, i.e. for all objects $\boldsymbol{e}$ and $\boldsymbol{e}^{\prime}$, the whole consisting of $\boldsymbol{e}$ and $\boldsymbol{e}^{\prime}$ is better than $\boldsymbol{e}$. In particular, 'self-concatenation' is valueincreasing, i.e. for all objects $\boldsymbol{e}$ and all $m>1$, $m \boldsymbol{e}$ is better than $(m-1) \boldsymbol{e}$. Now, we can define the relevant superiority relations corresponding to Griffin's trumping and discontinuity (I shall use Arrhenius's and Rabinowicz's terminology from now on):

Definition 1: An object $\boldsymbol{e}$ is superior to an object $\boldsymbol{e}^{\prime}$ if and only if, for all positive integers $n, \boldsymbol{e}$ is better than $n \boldsymbol{e}^{\prime}$.

Definition 2: An object $\boldsymbol{e}$ is weakly superior to an object $\boldsymbol{e}^{\prime}$ if and only if, for some positive integer $m$ and all positive integers $n, m e$ is better than $n \boldsymbol{e}^{\prime}$.

First, I shall assume that value is additive with respect to concatenation, i.e. the value of a concatenated whole is the sum of the value of each of its constituents. For this, the following condition is the principal necessary condition: ${ }^{13}$

Independence: An object $\boldsymbol{e}$ is at least as good as $\boldsymbol{e}^{\prime}$, if and only if $\boldsymbol{e}$ concatenated with any object is at least as good as $\boldsymbol{e}^{\prime}$ concatenated with that object. ${ }^{14}$

Hence, replacing $\boldsymbol{e}^{\prime}$ by $\boldsymbol{e}$ in any whole results in a whole that is at least as good.

Consider the Archimedean Condition that for all $\boldsymbol{e}, \boldsymbol{e}^{\prime}$ there exists a positive integer $n$ such that $n \boldsymbol{e}^{\prime}$ is at least as good as $\boldsymbol{e}$. This condition

${ }^{9}$ It is assumed that concatenation is associative, which means that we get the same whole from concatenating any three objects, regardless of the order in which they are concatenated.

10 That is: $\boldsymbol{e}$ is better than $\boldsymbol{e}^{\prime}$, if and only if $\boldsymbol{e}$ is at least as good as $\boldsymbol{e}^{\prime}$, and $\boldsymbol{e}^{\prime}$ is not as least as good as $\boldsymbol{e}$; and $\boldsymbol{e}$ is equivalent to $\boldsymbol{e}^{\prime}$ if and only if $\boldsymbol{e}$ is at least as good as $\boldsymbol{e}^{\prime}$, and $\boldsymbol{e}^{\prime}$ is at least as good as $\boldsymbol{e}$.

11 That is: for all objects $\boldsymbol{e}, \boldsymbol{e}^{\prime}, \boldsymbol{e}^{\prime \prime}:$ if $\boldsymbol{e}$ is at least as good as $\boldsymbol{e}^{\prime}$, and $\boldsymbol{e}^{\prime}$ is at least as good as $\boldsymbol{e}^{\prime \prime}$, then $\boldsymbol{e}$ is at least as good as $\boldsymbol{e}^{\prime \prime}$.

12 That is: for all objects $\boldsymbol{e}, \boldsymbol{e}^{\prime}$, either $\boldsymbol{e}$ is at least as good as $\boldsymbol{e}^{\prime}$ or $\boldsymbol{e}^{\prime}$ is at least as good as $\boldsymbol{e}$.

${ }^{13}$ Cf. David H. Krantz., R. Duncan Luce, Patrick Suppes and Amos Tversky, Foundations of Measurement, vol. 1: Additive and Polynomial Representations (San Diego, 1971), pp. 73-4.

14 Arrhenius and Rabinowicz only assume the 'only if' - part in their Independencecondition - that is all they need for their Observation 2. 
is called Archimedean because it corresponds to the Archimedean property of real numbers. Since the Archimedean property is true of the real numbers, the Archimedean Condition is necessary for measurement in real numbers. If the Archimedean condition holds and the domain is sufficiently rich to ensure the solvability condition that if $\boldsymbol{e}$ is better than $\boldsymbol{e}^{\prime}$, then there exists some $\boldsymbol{e}^{\prime \prime}$ such that $\boldsymbol{e}$ is equivalent to the whole consisting of $\boldsymbol{e}^{\prime}$ and $\boldsymbol{e}^{\prime \prime}$, the betterness relation could be represented by a real-valued function which is additive with respect to concatenation. ${ }^{15}$ Suppose that $\boldsymbol{e}$ is superior to $\boldsymbol{e}^{\prime}$ and Independence holds. Superiority violates the Archimedean Condition - superiority is precisely defined as the condition that there is no number such that $n \boldsymbol{e}^{\prime}$ is at least as good as $\boldsymbol{e}$. Consequently, the value ratio between $\boldsymbol{e}$ and $\boldsymbol{e}^{\prime}$ is infinite and cannot be measured by any real number. ${ }^{16}$

Consider next weak superiority. In his discussion of measurement of well-being, Griffin says about weak superiority (which he calls 'discontinuity') that it brings with it ${ }^{17}$

the suspension of addition; [...] we have a positive value that, no matter how often a certain amount is added to itself, cannot become greater than another positive value, and cannot, not because with piling up we get diminishing value or even disvalue (though there are such cases), but because [it is] the sort of value, that, even when remaining constant, cannot add up to some other value.

It is not part of the definition of weak superiority that value is additive. However, Griffin seems here to assume that it is. Weak superiority likewise violates the Archimedean Condition. However, weak superiority is further assumed to imply that the inferior value can add up to some amount of the weakly superior value. It is only when the amount of the weakly superior value is sufficiently large ('enough') that the inferior value cannot add up to this amount - addition is then 'suspended'. I shall demonstrate that this picture cannot be upheld. If $\boldsymbol{e}^{\prime}$ cannot add up to me, it cannot even add up to $\boldsymbol{e}$. In other words, if we assume Independence, then weak superiority collapses into superiority:

Observation 1: Independence implies that if some element $\boldsymbol{e}$ is weakly superior to another $\boldsymbol{e}^{\prime}$, then $\boldsymbol{e}$ is also superior to $\boldsymbol{e}^{\prime}$.

Proof: Assume, for reductio, that some element $\boldsymbol{e}$ is weakly superior to another element $\boldsymbol{e}^{\prime}$, but not superior to it. The fact that $\boldsymbol{e}$ is weakly superior to $\boldsymbol{e}^{\prime}$ means that there is some $m$, such that $m \boldsymbol{e}$ is better than any number of $\boldsymbol{e}^{\prime}$-elements. The fact that $\boldsymbol{e}$ is not superior to $\boldsymbol{e}^{\prime}$ means that there is some $q$, such that $\boldsymbol{e}$ is not better than $q \boldsymbol{e}^{\prime}$. Assume, for all $n=2,3, \ldots$, that $(n-1) \boldsymbol{e}$ is not better than $(n-1) q \boldsymbol{e}^{\prime}$. Independence

15 Krantz et al., Foundations of Measurement, vol. 1, p. 74 (Theorem 1).

16 Cf. Krantz et al., Foundations of Measurement, vol. 1, pp. 271-2.

17 Griffin, Well-Being, p. 85. 
then implies that $n \boldsymbol{e}$ is not better than $(n-1) q \boldsymbol{e}^{\prime}$ concatenated with $\boldsymbol{e}$. Since $\boldsymbol{e}$ is not better than qe, Independence implies that $(n-1) q \boldsymbol{e}^{\prime}$ concatenated with $\boldsymbol{e}$ is not better than $n q \boldsymbol{e}^{\prime}$. By mathematical induction, it then follows that $n \boldsymbol{e}$ is not better than $n q \boldsymbol{e}^{\prime}$. But then it cannot be the case that that there is some $m$, such that $m e$ is better than any number of $\boldsymbol{e}^{\prime}$-elements.

We know of course that if some element $\boldsymbol{e}$ is weakly superior to another element $\boldsymbol{e}^{\prime}$, then there is one object, namely me, that is superior to $\boldsymbol{e}^{\prime}$. However, Observation 1 implies also that $\boldsymbol{e}$ is superior to $\boldsymbol{e}^{\prime}$. This holds regardless of the value of $\boldsymbol{e}$ and regardless of the size of $m$. In other words, if we choose $\boldsymbol{e}$ to represent as small unit of value as we like, and let as large a number $m$ of these units as we like be superior to any number of a slightly smaller unit $\boldsymbol{e}^{\prime}$, the consequence, given Independence, will still be that just one unit of $\boldsymbol{e}$ will be better than any number of $\boldsymbol{e}^{\prime}$.

Arrhenius and Rabinowicz prove that, if in a decreasing sequence $\boldsymbol{e}_{1}, \ldots, \boldsymbol{e}_{n}$ the first element is superior to the last one, then Independence implies that some element in the sequence is superior to its immediate successor. ${ }^{18}$ So in this case we cannot come from the superior object to the inferior object through a number of steps where each object in the sequence is only marginally worse than its immediate predecessor. At least one step is itself a step to something drastically worse - as a matter of fact, we know from above that it is a step to something infinitely worse, since the difference cannot be measured by any real number.

\section{SUPERIORITY IN VALUE WHEN VALUE IS NON-ADDITIVE}

However, suppose we give up additivity, i.e. give up Independence. For this case, Arrhenius and Rabinowicz prove: for any two objects $\boldsymbol{e}$ and $\boldsymbol{e}^{\prime}$, where $\boldsymbol{e}$ is weakly superior to $\boldsymbol{e}^{\prime}$ without being superior to it, the domain must contain a finite decreasing sequence of objects in which the first element is superior to the last one, but no element is superior to its immediate successor. ${ }^{19}$

We know from Observation 1 that the requirement that weak superiority does not collapse into superiority is inconsistent with Independence. And as Arrhenius and Rabinowicz note, if Independence is denied, then it becomes possible that by concatenating some object $\boldsymbol{e}^{\prime}$ to itself any number of times, the marginal value of each contribution, though always positive, converges to zero, such that there is a finite upper limit to the aggregated value. I should like to demonstrate that this will necessarily be the case.

\footnotetext{
18 Arrhenius and Rabinowicz, 'Millian Superiorities', p. 134 (Observation 2). The proof is in Appendix 1, p. 138.

19 Arrhenius and Rabinowicz, 'Millian Superiorities', pp. 131-2 (Observation 1).
} 
Remember that we are dealing with a transitive and complete weak betterness relation defined on a countable set of objects on which a concatenation operation is defined, such that the domain is closed under concatenation. Since the domain is a countable set, the betterness relation can be represented ordinally by a real-valued function $V$, such that

$\boldsymbol{e}$ is at least as good as $\boldsymbol{e}^{\prime}$ if and only if $V(\boldsymbol{e}) \geq V\left(\boldsymbol{e}^{\prime}\right) .^{20}$

Now the following can be proved:

Observation 2: Suppose it is the case that some object $\boldsymbol{e}$ is weakly superior to another $\boldsymbol{e}^{\prime}$ without being superior to it. Then the sequence $V\left(\boldsymbol{e}^{\prime}\right), V\left(2 \boldsymbol{e}^{\prime}\right), V\left(3 \boldsymbol{e}^{\prime}\right), \ldots$ has an upper bound.

Proof: Since $\boldsymbol{e}$ is weakly superior to $\boldsymbol{e}^{\prime}$, there is some $m$, such that $V(m \boldsymbol{e})$ is greater than $V\left(n \boldsymbol{e}^{\prime}\right)$, no matter how big $n$ is. Assume, for reductio, that the sequence $V\left(\boldsymbol{e}^{\prime}\right), V\left(2 \boldsymbol{e}^{\prime}\right), V\left(3 \boldsymbol{e}^{\prime}\right), \ldots$ has no upper bound. We know that the sequence $V\left(\boldsymbol{e}^{\prime}\right), V\left(2 \boldsymbol{e}^{\prime}\right), V\left(3 \boldsymbol{e}^{\prime}\right), \ldots$ is increasing, because we have assumed that 'self-concatenation' is value-increasing. But any unbounded increasing infinite value sequence approaches infinity. Then there must be some $n$, such that $V\left(n \boldsymbol{e}^{\prime}\right)$ is greater than the finite value $V(m \boldsymbol{e})$, which contradicts that $\boldsymbol{e}$ is weakly superior to $\boldsymbol{e}^{\prime}$.

Hence, weak superiority is not a sign of a large difference between the superior and the inferior object, but rather dependent on how the value of self-concatenation of the inferior object develops. We also know ${ }^{21}$ that if some object $\boldsymbol{e}$ in a decreasing sequence is weakly superior to another $\boldsymbol{e}^{\prime}$ without being superior to it, then the domain also contains some object that is superior to $\boldsymbol{e}^{\prime}$. In fact, any object with a value above the upper bound of the $V\left(\boldsymbol{e}^{\prime}\right), V\left(2 \boldsymbol{e}^{\prime}\right), V\left(3 \boldsymbol{e}^{\prime}\right), \ldots$ will be superior to $\boldsymbol{e}^{\prime}$. Thus, under these circumstances, not even superiority is a sign of a radical difference.

It could even be the case that some object $\boldsymbol{e}$ is superior to $\boldsymbol{e}^{\prime}$, but not to some object $\boldsymbol{e}^{\prime \prime}$, which is worse than $\boldsymbol{e}^{\prime}$, because the aggregated value of self-concatenation of $\boldsymbol{e}^{\prime \prime}$ has a higher upper bound than that of $\boldsymbol{e}^{\prime}$ or even no upper bound and hence an aggregated value that could exceed the value of $\boldsymbol{e}$. Note also that if self-concatenation of $\boldsymbol{e}^{\prime \prime}$ has a higher upper bound than that of $\boldsymbol{e}^{\prime}$, then we have a case where $\boldsymbol{e}^{\prime \prime}$ is weakly superior to $\boldsymbol{e}^{\prime}$ even though $\boldsymbol{e}^{\prime \prime}$ is worse than $\boldsymbol{e}^{\prime}$.

Arrhenius and Rabinowicz also prove that if in a finite sequence of objects the first element is weakly superior to the last element, then there exists at least one element that is weakly superior to its

20 Cf. Krantz et al., Foundations of Measurement, vol. 1, p. 39 (Theorem 2.1).

21 From Arrhenius and Rabinowicz, 'Millian Superiorities', p. 131 (Observation 1). 
immediate successor. ${ }^{22}$ Given my observations above, there is a simple and informative proof of this observation:

Proof: Suppose Independence is fulfilled. Because of my Observation 1 , weak superiority collapses into superiority and we know ${ }^{23}$ that in a decreasing sequence where the first element is superior (and therefore also weakly superior) to the last element, then some element in sequence is superior (and therefore also weakly superior) to its immediate successor. And if Independence is not fulfilled, we know from my Observation 2 that the last element, concatenated by an element like itself any number of times, has an upper bound. The preceding element, concatenated any number of times by an element like itself, either has an upper bound which is higher than this or has no upper bound, in which case it is weakly superior to the last element (because there will be some number of this element which is better than any number of the last) and the proof is done, or it has an upper bound that is lower or equal to the bound of the last element, in which case it is not weakly superior to it (indeed, if the upper bound is lower the last element would be weakly superior to the preceding one). In the latter case, we can repeat the procedure until we are either done or finally reach the next-to-the-first element, which in that case must have an upper bound that is lower than or equal to the bound of the last element; but since the first element by hypothesis is weakly superior to the last one, it is also weakly superior to this one.

Arrhenius and Rabinowicz are somewhat surprised by their result, because they start out with the intuition that both superiority and weak superiority are drastic differences in value. Whereas Rabinowicz is willing to accept that an element can be weakly superior to another even though it is only marginally better, Arrhenius ${ }^{24}$ sticks to the intuition and takes the line that Rabinowicz's and his results provide an argument against superiority and weak superiority in all contexts where it is possible to construct a sequence of objects in which the value differences between adjacent objects are marginal.

But as my results show, weak superiority does not depend on the difference between elements, but solely on how the aggregated value of self-concatenation develops. Hence, even in a decreasing finite sequence in which each consecutive element is only marginally worse than the immediately preceding one, weak superiority can obtain; and even an element which is worse than another might be weakly superior to it. For the same reason, weak superiority between the extrema of a finite

22 Arrhenius and Rabinowicz, 'Millian Superiorities', pp. 136-7 (Observation 3).

${ }_{23}$ From Arrhenius and Rabinowicz, 'Millian Superiorities', p. 134 (Observation 2).

${ }^{24}$ See here also Gustaf Arrhenius, 'Superiority in Value', Philosophical Studies 123 (2005), pp. 97-114. 
sequence does not mean that the last element is radically worse than the point of departure. Moreover, if we have a finite decreasing sequence of objects in which the first element is superior to the last one, and some element in the sequence is weakly superior to another one, then not even superiority is a radical difference in value.

Arrhenius further claims that superiority and weak superiority share some problems when we consider outcomes that involve both superior and inferior objects. ${ }^{25}$ Consider three objects $\boldsymbol{e}, \boldsymbol{e}^{\prime}$ and $\boldsymbol{e}^{\prime \prime}$, where $\boldsymbol{e}$ is only marginally better than $\boldsymbol{e}^{\prime}$, and $\boldsymbol{e}^{\prime}$ is clearly better than $\boldsymbol{e}^{\prime \prime}$. Assume further that $\boldsymbol{e}$ is superior or weakly superior to $\boldsymbol{e}^{\prime}$ (and in the latter case, let $n$ be a number such that $n \boldsymbol{e}$ is better than any number $\boldsymbol{e}^{\prime}$ ). Compare a whole, $\boldsymbol{a}$, consisting of $n \boldsymbol{e}$, with another whole, $\boldsymbol{b}$, consisting of $n \boldsymbol{e}$ and $m \boldsymbol{e}^{\prime \prime}$, where $m$ is much greater than $n$. By the assumption of value increasingness, $\boldsymbol{b}$ is better than $\boldsymbol{a}$. Consider now a third whole, $\boldsymbol{c}$, consisting of $(n+m) \boldsymbol{e}^{\prime}$. Since $\boldsymbol{e}$ (or $n \boldsymbol{e}$ ) is superior to $\boldsymbol{e}^{\prime}, \boldsymbol{a}$ is better than $\boldsymbol{c}$. However, Arrhenius says, compare $\boldsymbol{b}$ and $\boldsymbol{c}$ : since the loss of getting $\boldsymbol{e}^{\prime}$ instead of $\boldsymbol{e}$ is only marginal, and the gain from getting $\boldsymbol{e}^{\prime}$ instead of $\boldsymbol{e}^{\prime \prime}$ is bigger, there should be some sufficiently large $m$, such that $\boldsymbol{c}$ is better than $\boldsymbol{b}$; and then, by transitivity, we would have that $\boldsymbol{c}$ is better than $\boldsymbol{a}$. Hence, the notions of superiority and weak superiority seem to imply a contradiction in this case.

But, taken at face value, Arrhenius's reasoning is mistaken. If $\boldsymbol{e}$ is superior (or weakly superior) to $\boldsymbol{e}^{\prime}$, then the loss of $\boldsymbol{e}$ (or the loss of having less than $n \boldsymbol{e}$ ) cannot be compensated by any number of $\boldsymbol{e}^{\prime}$. Therefore, $\boldsymbol{b}$ is better than $\boldsymbol{c}$, and there is no contradiction. When he says that there is marginal loss for each $\boldsymbol{e}$-object that has been exchanged for an $\boldsymbol{e}^{\prime}$ object, but a bigger gain for each $\boldsymbol{e}^{\prime \prime}$-object that has been exchanged for an $\boldsymbol{e}^{\prime}$-object, he appears to assume Independence. But if Independence is fulfilled, and $\boldsymbol{e}$ is superior to $\boldsymbol{e}^{\prime}$, the value difference between $\boldsymbol{e}$ and $\boldsymbol{e}^{\prime}$ cannot be marginal, which it is by hypothesis in the example.

However, Arrhenius might still have a point. When he says that it is hard to deny that there is some $m$ such that the smaller number of smaller losses is compensated for by the greater number of greater gains, he could be understood as implying that, given the marginal difference between $\boldsymbol{e}$ and $\boldsymbol{e}^{\prime}$, it is implausible that the loss of $n \boldsymbol{e}$ (or even $\boldsymbol{e}$ ) cannot be compensated by a sufficiently large number $m$ of $\boldsymbol{e}^{\prime}$ objects; otherwise, the marginal value of adding extra $\boldsymbol{e}^{\prime}$-objects would implausibly - diminish extremely rapidly. This may be true, in which case $\boldsymbol{c}$ would be better than $\boldsymbol{b}$ (which is better than $\boldsymbol{a}$ ); but if it is, $\boldsymbol{e}$ cannot be superior or weakly superior to $\boldsymbol{e}^{\prime}$, and so $\boldsymbol{a}$ cannot at the same time be better than $\boldsymbol{c}$. Thus, even though there is no contradiction, this

${ }^{25}$ Arrhenius, 'Superiority in Value', pp. 108-9. 
reasoning could support his initial claim that superiority relations are implausible in these contexts.

\section{AN EXAMPLE OF LARRY TEMKIN'S}

It is illuminating to apply these results on an example of Larry Temkin's. ${ }^{26}$ Temkin asks us to consider the following claims: ${ }^{27}$

Claim 1: for any unpleasant or 'negative' experience, no matter what the intensity and duration of that experience, it would be better to have that experience than one that was only a little less intense but twice as long.

Claim 2: there is a continuum of unpleasant or 'negative' experiences ranging in intensity, for example, from extreme forms of torture to the mild discomfort of a hangnail.

Claim 3: a mild discomfort for the duration of one's life would be preferable to two years of excruciating torture, no matter the length of one's life.

Temkin then argues that, taken together, these claims constitute a serious threat to the transitivity of the betterness relation.

Suppose we have a sequence of negative experiences for the duration of one year, $\boldsymbol{a}, \boldsymbol{b}, \ldots, \boldsymbol{y}$, of descending intensity ranging from excruciating torture to the discomfort of a hangnail. Suppose further that negative experiences can be concatenated with each other, including the possibility of self-concatenating, and that concatenation is negatively value increasing. Then consider the sequence $2 \boldsymbol{a}$ (two years of excruciating torture), $4 \boldsymbol{b}$ (four years of slightly less intense torture), .., $2 n \boldsymbol{y}$ (the discomfort of a hangnail for very many years). It follows from the Claim 1 that $2 \boldsymbol{a}$ is better than $4 \boldsymbol{b}, 4 \boldsymbol{b}$ is better than $8 \boldsymbol{c}$ and so on down to $2(n-1) \boldsymbol{x}$ is better than $2 n \boldsymbol{y}$. Transitivity then implies that $2 \boldsymbol{a}$ is better than $2 n \boldsymbol{y}$, but Claim 3 says that $2 n \boldsymbol{y}$ is better than $2 \boldsymbol{a}$, thus violating transitivity.

In his defence of Claim 3, Temkin appears to refer to some kind superiority relation: ${ }^{28}$

It is commonly observed that many people suffering a slight headache does not add up to a single person suffering a migraine. This observation reflects an important fact. [...] pains and pleasures are not like pennies and grams. They simply do not add up in the way they would have to for us to reject claim 3.

${ }^{26}$ Larry S. Temkin, 'A Continuum Argument for Intransitivity', Philosophy \& Public Affairs 25-3 (1996), 174-210.

27 Temkin, 'A Continuum Argument for Intransitivity', p. 179.

28 Temkin, 'A Continuum Argument for Intransitivity', p. 190. 
More precisely, he appears to apply a version of weak superiority: ${ }^{29}$

But, in essence, I think significant amounts of torture have lexical priority over any amount of a hangnail, [...]. My model for this is something like the following. Torture's badness might range from 0 to 10, depending on its duration, with two years of torture being, say, a 7. A hangnail's badness might range from 0 to 1 . Prolonging a hangnail increases the value of the decimal places representing its 'badness score,' but the fundamental gap between 1 and 7 is never affected.

It will be convenient to assume the existence of a worseness relation, is at least as bad as -, defined on the domain of negative experiences by $\boldsymbol{e}$ is at least as bad as $\boldsymbol{e}^{\prime}$ if and only if $\boldsymbol{e}^{\prime}$ is as least as good as $\boldsymbol{e}$. Let superiority and weak superiority relations be defined for worseness: an object $\boldsymbol{e}$ is superior in worseness to an object $\boldsymbol{e}^{\prime}$ if and only if, for all positive integers $n, \boldsymbol{e}$ is worse than $n \boldsymbol{e}^{\prime}$; and an object $\boldsymbol{e}$ is weakly superior in worseness to an object $\boldsymbol{e}^{\prime}$ if and only if, for some positive integer $m$ and all positive integers $n, m \boldsymbol{e}$ is worse than $n \boldsymbol{e}^{\prime}$.

Suppose, first, that the value of pain is additive in the sense that it is measured by adding up the intensity for each year. Then Independence is fulfilled. Since the intensity in Temkin's example is the same throughout the duration of the negative experience, the value is simply measured by the intensity multiplied by the duration.

We know from Observation 1 that if some duration of torture with intensity $\boldsymbol{a}$ is worse than any duration of a hangnail with intensity $\boldsymbol{y}$, then any duration of torture with intensity $\boldsymbol{a}$, however short, is worse than any duration of a hangnail with intensity $\boldsymbol{y}$. And we know ${ }^{30}$ that some experience in the sequence is superior in worseness to its immediate successor. Then claim 1 is false, since the successor at this place in the sequence cannot add up to its predecessor; and also claim 2 will be false, since there will be discontinuity in the sequence at this place.

In case there is just one such discontinuity in the sequence, say at the $i$ th step between $\boldsymbol{p}$ and $\boldsymbol{q}$, and claim 1 holds for adjacent pairs above and below this step, the picture we get is one sequence where $2 \boldsymbol{a}$ is better than $4 \boldsymbol{b}, 4 \boldsymbol{b}$ is better than $8 \boldsymbol{c}$, and so on down to $2 i \boldsymbol{p}$; on a second order of magnitude below this sequence, there is another sequence where $2(i+1) \boldsymbol{q}$ is better than $2(i+2) \boldsymbol{r}$ and so on down to $2 n \boldsymbol{y}$. Hence, perhaps surprisingly, the best object is $2(i+1) \boldsymbol{q}$ and the worst is $2 i \boldsymbol{p}$, but there is no intransitivity.

Suppose next that that Independence does not hold. We then know from Observation 2 that if torture with intensity $\boldsymbol{a}$ is superior in worseness to any duration of a hangnail with intensity $\boldsymbol{y}$ without

29 Temkin, 'A Continuum Argument for Intransitivity', p. 191.

${ }^{30}$ From Arrhenius and Rabinowicz, 'Millian Superiorities', p. 134 (Observation 2). 
being superior in worseness to it, then the badness of the discomfort from a hangnail for a year, concatenated with itself over time, has an upper limit. We also $\mathrm{know}^{31}$ that there exists at least one experience in the sequence that is weakly superior in worseness to its immediate successor.

In this case, claim 2 is true. However, supposing that there is just one case of weak superiority in worseness between adjacent experiences in the sequence, say at the $i$ th step between $\boldsymbol{p}$ and $\boldsymbol{q}$, claim 1 is false for this step, because some duration of $\boldsymbol{p}$ will be worse than any duration of $\boldsymbol{q}$. In fact, it can be shown that $2 i \boldsymbol{p}$ is worse than any duration $\boldsymbol{q}$. It is assumed that $2 \boldsymbol{a}$ is worse than any duration of $\boldsymbol{y}$. Since it is also assumed that none of the first $i$ experiences is weakly superior to its successor, we also assume that $2 \boldsymbol{a}$ is better than $4 \boldsymbol{b}, 4 \boldsymbol{b}$ is better than $8 \boldsymbol{c}$, and so on down to $2 i \boldsymbol{p}$, and hence, that $2 i \boldsymbol{p}$ is worse than any duration of $\boldsymbol{y}$. It is clear from my proof of Arrhenius's and Rabinowicz's Observation 3 that in the sequence from $\boldsymbol{x}$ up to $\boldsymbol{q}$, the badness of each experience, concatenated with itself over time, will have an upper limit which is not worse than the limit of its predecessor. We might therefore conclude that any duration of $\boldsymbol{q}$ has an upper limit, which is not worse than $2 i \boldsymbol{p}$. Hence, we get the picture that $2(i+1) \boldsymbol{q}$ is better than $2 \boldsymbol{a}$, $2 \boldsymbol{a}$ is better than $4 \boldsymbol{b}, 4 \boldsymbol{b}$ is better than $8 \boldsymbol{c}$, and so on down to $2 i \boldsymbol{p}$. The experiences $2(i+1) \boldsymbol{q}, 2(i+2) \boldsymbol{r}, \ldots, 2 n \boldsymbol{y}$ are all better than $2 \boldsymbol{a}$; however, the example is not sufficiently detailed to determine their pairwise ranking as regards worseness. For low durations, an experience in the sequence might be better than its successor, but for long durations where the badness approaches its upper limit, the successor might be worse than the predecessor. One way or the other, however, there is no reason to suspect intransitivity.

The common point is that, in order for claim 1 to be generally true, pain has to be aggregated additively with respect to time in a way that fulfils the Archimedean Condition. However, in his defence of claim 3, Temkin denies that pain is aggregated in this way, because otherwise claim 3 would be false. Temkin might of course object that my reasoning presupposes transitivity. But in order to represent a case against transitivity, each of the three claims should be plausible in its own right. If Temkin implicitly appeals to two conflicting notions of aggregation, we have good reason to doubt that the three claims are simultaneously true. I shall not here attempt to decide which form of aggregation is correct. However, I should like to mention that if aggregation of the disvalue of pain over time implies diminishing marginal disvalue, this does not necessarily transfer to interpersonal

31 From Arrhenius and Rabinowicz, 'Millian Superiorities', p. 136 (Observation 3). 
aggregation of the disvalue of pain, as Temkin hints at in the first quotation above. The idea that intrapersonal aggregation transfers to interpersonal aggregation is at the heart of Griffin's suggestion, to which I turn next.

\section{SUPERIORITY IN VALUE AND THE REPUGNANT CONCLUSION IN A CONTEXT WHERE VALUE IS ADDITIVE}

Griffin's main idea is that no amount of certain less important values can ever compensate a substantial loss of certain more important and genuine values. The underlying picture here is that welfare depends on the degree to which a number of prudential values are realized. ${ }^{32}$ More precisely, I shall assume that welfare is measured by the sum of the value contribution from each value. And if one of the important values is realized to a sufficient degree, its value contribution is such that the contribution from an unimportant value never can add up to it, no matter how much it is realized.

Griffin gives an example, in which an unimportant value is a sort of residual value to an important one, such that when the latter is lost, we might get the former: ${ }^{33}$ the important value is 'appreciation of beauty'. If we gradually reduce the degree to which this value is realized, we shall eventually reach a point at which it is lost. We might instead have 'kicks of kitsch', but they are different, that is, they represent a different value, which still gives a positive contribution but one that is inferior to the contribution from genuine appreciation of beauty.

Griffin's suggestion is based on the idea that weak superiority between valuable objects is a more plausible condition than superiority. But since his suggestion also implies a context where value is additive, we should expect from Observation 1 that weak superiority collapses into superiority. However, to be able to apply Observation 1 in this context, I need to set up a slightly more complicated apparatus.

I assume a list of prudential values $A, B, C, \ldots$ In a given life, each of these values is realized to a certain degree which I shall assume can be measured by a non-negative number. Thus, there is a domain of possible lives, $\boldsymbol{L}=\left\{\boldsymbol{l}_{1}, \boldsymbol{l}_{2}, \boldsymbol{l}_{3}, \ldots\right\}$, where each life is represented by a vector $(a, b, c, \ldots)$ and $a, b, c, \ldots$ are non-negative numbers describing the degree to which each of the values $A, B, C, \ldots$ is realized in this life.

As before, there is a weak betterness relation, - is at least as good as -, defined on this domain, which is assumed to be transitive and complete. This relation embodies global preferences over the domain of possible lives, which Griffin considers basic for the measurement of welfare.

32 Cf. the list in Griffin, Well-Being, p. 67.

33 Griffin, Well-Being, pp. 86-7. 
Some values might really be disvalues, giving a negative contribution to overall welfare. In this context, I shall only consider values giving positive or zero contribution to overall welfare. However, I shall allow for the fact that the marginal contribution from each positive value diminishes the more it is realized.

Next, I shall assume some necessary conditions for value contributions to be additive, such that the welfare of a life, $w(\boldsymbol{l})$ where $\boldsymbol{l}=(a, b, c, \ldots)$, could be measured by the sum of contributions $w_{A}(a)+w_{B}(b)+w_{C}(c)+\ldots$ from each of the values. Here, I draw on what is known as additive conjoint measurement. ${ }^{34}$ Additive conjoint measurement does not rely on a simple concatenation procedure like the one outlined above, with respect to which it is additive. In order to establish additivity, it simulates concatenation in a more complicated way. ${ }^{35}$ The first necessary condition is a form of independence known as strong separability:

Definition 3: Consider some subset of values, say $P$ and $Q$, and let the degree of realization of each of the remaining values be kept constant $(\underline{a}, \underline{b}, \ldots, \underline{o}, \underline{r}, \underline{s}, \ldots)$. The betterness relation will rank alternative combinations $(p, q)$ of the degree to which $P$ and $Q$ are realized, given this fixed choice of the degree of realization of the remaining values. If this ranking is the same for all possible fixed choices, the subset of values $P$ and $Q$ are said to be separable in the betterness relation. If any arbitrary subset of values is separable in this way, the betterness relation is said to be strongly separable.

If the betterness relation is strongly separable, it induces a transitive and complete betterness relation on each subset of values. In other words, we can then evaluate the betterness of each subset of values independently from the other values.

The other necessary condition I shall introduce is the existence of standard sequences for each value. A standard sequence defines value differences having non-zero, equal spacing in the intended numerical representation of the value contribution from each value. Consider again the values $P$ and $Q$. Arbitrarily, define some unit $q_{1}$ on $Q$. Now, find some degree $p_{1}$, such that $\left(p_{1}, 0\right)=\left(0, q_{1}\right) \cdot{ }^{36}$ (Note that this only works if $P$ and $Q$ are both important or both unimportant. Hence, I

${ }^{34}$ Cf. Krantz et al., Foundations of Measurement, vol. 1, pp. 245-315 (ch. 6).

35 In the standard framework, it is assumed that the set of possible lives, $\boldsymbol{L}$, is a product set. This means that the values in a life are independently realizable, i.e. that that the domain contains every possible combination of degrees of realization of values. However, this is not a condition which is necessary for the additive representation as such.

36 To be sure this can be done, we need to assume a solvability condition, cf. Krantz et al., Foundations of Measurement, vol. 1, p. 301. This is another structural condition, which is not necessary for the additive representation. 
shall assume that there are at least two values on each level.) Then, find some degree $p_{2}$, such that $\left(p_{2}, 0\right)$ is equivalent with $\left(p_{1}, q_{1}\right)$. Go on and find some degree $p_{3}$, such that $\left(p_{3}, 0\right)$ is equivalent with $\left(p_{2}, q_{1}\right)$. Similarly, define $p_{4}, p_{5}, \ldots$ Now, we have defined a standard sequence on value $P$ :

Definition 4: A sequence of degrees $p_{i}, p_{i+1}, \ldots, i=1,2, \ldots$, of some value $P$ is a standard sequence if and only if there exists $q_{1}, q_{2}$ on some other value $Q$ such that $q_{1}$ is better than $q_{2}$ and for all $i=1,2, \ldots,\left(p_{i}\right.$, $\left.q_{1}\right)$ is equally as good as $\left(p_{i+1}, q_{2}\right)$. A standard sequence can be either finite or infinite.

A similar procedure can be used on $Q$. And we can go on adding definitions of fractions of the chosen unit.

The plausibility of these conditions depends of course a lot on whether it is possible to describe and individuate values in a way such that the contribution of any subset of values, as derived from our basic preferences over possible lives, is independent of the degree to which other values are realized. I shall not here attempt to argue that these conditions are in fact fulfilled. The point is merely to point out that these conditions are necessary for the argument Griffin wants to make, and to spell out the consequences.

The standard sequence $p_{1}, p_{2}, p_{3}, \ldots$ can be understood as additive self-concatenation of $p_{1}$, where $p_{2}$ is a whole consisting of $p_{1}$ concatenated with an object like itself, and where also $q_{1}$ is defined as an object like $p_{1}$. And given strong separability and a standard sequence $p_{1}, p_{2}, p_{3}, \ldots$ defined on $P$ using $q_{1}, q_{2}, q_{3}, \ldots$ on $Q$, it follows straightforwardly from the definition of standard sequences that Independence will be fulfilled for this standard sequence on $P$, such that, for all non-negative integers $n, n^{\prime}$ and $m$ where $n \geq n^{\prime}, p_{n}$ is at least as good as $p_{n^{\prime}}$ if and only if $p_{n+m}$ is at least as good as $p_{n^{\prime}+m} \cdot{ }^{37}$

Suppose now, as Griffin suggests, that $A$ is weakly superior to $B$ in the sense that, for any standard sequences $a_{1}, a_{2}, a_{3}, \ldots$ and $b_{1}, b_{2}$, $b_{3}, \ldots,{ }^{38}$ there is some $m$ such that $\left(a_{m}, 0\right)$ is better than $\left(0, b_{n}\right)$, no matter how big $n$ is. In the context of additive conjoint measurement, the Archimedean condition can be stated thus: every strictly bounded standard sequence is finite. ${ }^{39}$ But as weak superiority is defined here, it violates the Archimedean condition, because the standard sequence $b_{1}, b_{2}, b_{3}, \ldots$ is infinite and still strictly bounded by $\left(a_{m}, 0\right)$. Hence, the

37 In other words, if a numerical representation were possible (which I have not yet assumed), we would have $w_{P}\left(p_{2}\right)=2 w_{P}\left(p_{1}\right), w_{P}\left(p_{3}\right)=3 w_{P}\left(p_{1}\right), \ldots$

${ }_{38}$ Note that these standard sequences are not defined relative to each other.

39 Cf. Krantz et al., Foundations of Measurement, vol. 1, p. 253. 
difference between $a_{m}$ and $b_{1}$ is infinitely large and cannot be measured by any real number. This is precisely what Griffin needs.

However, we are now in a position where we can apply Observation 1 and demonstrate that weak superiority collapses into superiority: if $\left(a_{m}, 0\right)$ is better than $\left(0, b_{n}\right)$, no matter how big $n$ is, then it follows from Observation 1 that $\left(a_{1}, 0\right)$ is better than $\left(0, b_{n}\right)$, no matter how big $n$ is. In other words, even the smallest degree to which $A$ can be realized will be better than $B$, no matter the degree to which it is realized. Consequently, contrary to his inclination, if Griffin wants to keep the idea that some levels of welfare do not add up to others, he is bound to base his argument on superiority rather than on weak superiority.

Even so, we might still have a credible view which might avoid the Repugnant Conclusion. I shall make some of its implications clear. Consider a sequence of lives with decreasing of levels of welfare $\boldsymbol{a}$, $\boldsymbol{b}, \boldsymbol{c} \ldots, \boldsymbol{z}$. Given that we accept the Utilitarian Total Principle, a concatenation procedure is defined, the betterness relation is given and the Independence condition is satisfied. Griffin suggests that some number of people $n$ at some level, say $\boldsymbol{m}$, is weakly superior to the level $\boldsymbol{z}$. But then it follows from Observation 1, first, that $\boldsymbol{m}$ is superior to $\boldsymbol{z}$; in other words, it would be the case that even one person at $\boldsymbol{m}$ outranks any number of lives at $\boldsymbol{z}$.

Furthermore, we know ${ }^{40}$ that if we have a decreasing sequence $\boldsymbol{m}$, $\boldsymbol{n}, \ldots, \boldsymbol{z}$ where $\boldsymbol{m}$ is superior to $\boldsymbol{z}$, then there will be some level in the sequence which is superior to its immediate successor. In other words, one person living at the lowest level before the discontinuity sets in is superior to any number of people living at $\boldsymbol{z}$. Call this level $\boldsymbol{y}$. Suppose there is some $n$ such that $n \boldsymbol{y}$ is equally good as $\boldsymbol{m}$. Then the same consequence would follow from my Observation 1: since $\boldsymbol{y}$ is weakly superior to $\boldsymbol{z}$ (because $\boldsymbol{m}$ and therefore also $n \boldsymbol{y}$ is superior to $\boldsymbol{z}$ ), it follows that $\boldsymbol{y}$ is superior to $\boldsymbol{z}$.

Strictly speaking, this view does not avoid the Repugnant Conclusion as I have stated it. I shall assume that welfare is measurable on a scale that has an extension of the real numbers with infinitesimal numbers. ${ }^{41}$ I shall not go into technical details about infinitesimal numbers. ${ }^{42}$ It suffices with the intuitive understanding that adding an infinitesimal number to another infinitesimal number results in

${ }^{40}$ From Arrhenius and Rabinowicz, 'Millian Superiorities', p. 134 (Observation 2).

41 Alternatively, we could imagine that welfare is measured on two dimensions, cf. M. Hausner: 'Multidimensional Utilities', Decision Processes, ed. R. M. Thrall, C. H. Coombs and R. L. Davis (New York, 1954), pp. 167-80.

${ }_{42}$ There is a rigorous treatment in Abraham Robinson, Non-Standard Analysis, rev. edn. (Amsterdam: North-Holland, 1974). As for measurement, see Louis Narens, 'Measurement without Archimedean Axioms', Philosophy of Science 41 (1974), pp. 374-93; Louis Narens, 'Minimal Conditions for Additive Conjoint Measurement and 
an infinitesimal number, and consequently, that multiplication of an infinitesimal number with an integer results in another infinitesimal number. On this scale, it is still the case that, for any positive finite level of welfare, $\boldsymbol{y}$, however close to zero, a population of $m$ people at $\boldsymbol{y}$ is better than $n$ people at $\boldsymbol{a}$, provided $m \boldsymbol{y}>n \boldsymbol{a}$. It is just that below such level $\boldsymbol{y}$, however low, there are lives definitely worse than $\boldsymbol{y}$ but still worth living - these are the ones measured by infinitesimally small levels. So maybe the level $\boldsymbol{y}$ is not so bad after all. ${ }^{43}$

Whether this is a credible view depends on how the zero on the scale is determined, ${ }^{44}$ and where the discontinuity sets in. Like Parfit, Griffin appears to have a zero for the scale of welfare in mind, which is something like the level where there is no point of living the life, a life with neither positive nor negative value at all ${ }^{45}$ and implicitly, this zero is also the level on which the existence of the person is indifferent from the point of view of the Total Principle. ${ }^{46}$

As regards the discontinuity, we saw above that even the smallest degree to which some superior value $A$ can be realized will be better than some inferior value $B$, no matter the degree to which it is realized. This means that a life in which just one superior value is realized to the smallest possible degree is infinitely better that a life in which no superior values are realized. Interestingly, Griffin seems to accept this consequence, when he says: ${ }^{47}$

Then we might wish to stop the slide [...] at that point along the line where people's capacity to appreciate beauty, to form deep loving relationships, to accomplish something with their lives beyond just staying alive...all disappear.

Given this more explicit statement, we can now evaluate the repugnantlike conclusion: for any positive finite (i.e. non-infinitesimal) level of welfare, $\boldsymbol{y}$, however low, a population of $m$ people at $\boldsymbol{y}$ is better than $n$ people at $\boldsymbol{a}$, provided $m \boldsymbol{y}>n \boldsymbol{a}$. It means that a sufficiently large number of persons with lives just barely realizing one important value in life will represent a greater total and therefore be better than some number of persons with a very high welfare. This still seems to me a rather repugnant conclusion.

Qualitative Probability', Journal of Mathematical Psychology 11 (1974), pp. 404-30; and

Heinz J. Skala, Non-Archimedean Utility Theory (Dordrecht, 1975).

${ }_{43}$ I owe this interpretation to a communication from John Broome.

${ }^{44}$ Cf. John Broome, Weighing Lives (Oxford, 2004), p. 138. This point is largely overlooked.

${ }^{45}$ Griffin, Well-Being, pp. 130-1, 345 (n. 12).

${ }^{46}$ Conceptually, however, these are two different questions. Cf. Broome, Weighing Lives, pp. pp. 199-214 (ch. 14).

${ }^{47}$ Griffin, Well-Being, p. 340 (n. 27), my italics. 
Another troublesome implication stems from the fact that even one person living at a finite level as close to zero as we want is better than any number of people living at a positive infinitesimal level. It means that the view implies a version of what Parfit calls the Absurd Conclusion: it is better that there live no people at all than a number of people with infinitesimally low welfare and one person in suffering at any finite negative level. Like Parfit, many would find this implication absurd.

\section{SUPERIORITY IN VALUE AND THE REPUGNANT CONCLUSION IN A CONTEXT WHERE VALUE IS NON-ADDITIVE}

Suppose next that we give up the conditions necessary for additivity, i.e. strong separability and the existence of standard sequences. There is a transitive and complete weak betterness relation defined on the domain of possible lives, $\boldsymbol{L}=\left\{\boldsymbol{l}_{1}, \boldsymbol{l}_{2}, \boldsymbol{l}_{3}, \ldots\right\}$, where each life is represented by a vector $(a, b, c, \ldots)$ and $a, b, c, \ldots$ are non-negative numbers describing the degree to which each of the values $A, B, C, \ldots$ is realized in this life. Assume that weak betterness fulfils strong monotonicity such that, for all pairs of lives, if one life has all values realized to at least the same degree as another, and at least one value is realized to higher degree, then it is better.

If in this framework $A$ is weakly superior to $B$, it means that there is some $\underline{a}$ such that if $A$ drops below $\underline{a}$, no degree of $B$ can ever compensate this loss in value (other values kept constant). However, this is compatible with the superior and the inferior life being measurable on the same real-valued scale. If we were to apply the Utilitarian Total Principle, ${ }^{48}$ it is clear that $n$ people on the superior level always can be outweighed by a sufficiently large number $m$ on the inferior level. Hence, in this case, the Repugnant Conclusion could not be avoided.

However, even though Griffin's suggestion appears to be based on the idea that values are additive, he is in fact rather sceptical about measuring welfare on a cardinal scale. ${ }^{49}$ Thus, it is possible to understand how weak superiority between prudential values would get transferred to interpersonal calculation in another way. Just as the comparison of lives is a matter of basic preferences, not of calculations based on other sources, comparisons of populations will have to be

\footnotetext{
${ }^{48}$ However, in this case, the framework described so far does not provide a cardinal scale that would allow summing up welfare.

49 Cf. Griffin, Well-Being, pp. 88, 98-102.
} 
a matter of basic rankings. ${ }^{50}$ If this is the case, there is no route to determining the most beneficent outcome through simple summation. Then Griffin's suggestion, 'Perhaps it is better to have a certain number of people at a certain high level than a very much larger number at a level where life is just worth living', could be interpreted as such a basic ranking where the weak superiority does not collapse.

Consider a sequence of lives with decreasing of levels of welfare $\boldsymbol{a}$, $\boldsymbol{b}, \boldsymbol{c} \ldots, \boldsymbol{z}$. I assume that there is a concatenation procedure, such that we can concatenate lives into wholes (that is, populations) and that the domain is closed under concatenation. I also assume that a weak betterness relation, which is transitive and complete, is defined on the domain. In this framework, the ranking can be stated as: there is some level $\boldsymbol{p}$, which is weakly superior to $\boldsymbol{z}$, without being superior to it. This means that there is some number $m$ such that, for all positive integers $n, m \boldsymbol{p}$ is better than $n \boldsymbol{z}$.

We know from section 1 that the betterness relation on this domain can be represented ordinally by a real-valued function, $V$. And we know from Observation 2 that if $\boldsymbol{p}$ is weakly superior to $\boldsymbol{z}$ without being superior to it, then the increasing sequence $V(\boldsymbol{z}), V(2 \boldsymbol{z}), V(3 \boldsymbol{z}), \ldots$ has an upper bound.

Thus, the basic ranking in this context implies that adding more people at $\boldsymbol{z}$ has diminishing marginal value that converges to zero. It makes the value of a person at $\boldsymbol{z}$ depend on how many other people there are at this level. And this is implausible. I can think of no reason having to do with beneficence why one out of two persons at the same level of welfare should have more weight than the other. This is my main objection to this view.

Arrhenius states another objection, ${ }^{51}$ namely that this view either implies the Mere Addition Paradox ${ }^{52}$ or else it violates what he calls The Inequality Aversion Condition: for any triplet of welfare levels $\boldsymbol{a}$, $\boldsymbol{y}, \boldsymbol{z}$ and for any population $n \boldsymbol{a}$, there is some number $m$, such that the perfectly equal population $(m+n) \boldsymbol{y}$ is at least as good as the population combined of $n \boldsymbol{a}$ and $m \boldsymbol{z}$. The combined population $n \boldsymbol{a}$ and $m \boldsymbol{z}$ is better than the population $n \boldsymbol{a}$. If we assume that the view complies with The Inequality Aversion Condition, the equal population $(n+m) \boldsymbol{y}$ will be at least as good as the population combined of $n \boldsymbol{a}$ and $m \boldsymbol{z}$. By transitivity, the equal population $(m+n) \boldsymbol{y}$ is then at least as good as $n \boldsymbol{a}$. But the view is supposed to imply that $n \boldsymbol{a}$ is better than $(m+n) \boldsymbol{y}$ in the cases

50 This is how Gustaf Arrhenius: Future Generations. A Challenge for Moral Theory (Uppsala, 2000), pp. 96-7, understands Griffin. The interpretation is also apparent in Roger Crisp, 'Utilitarianism and the Life of Virtue', The Philosophical Quarterly 42 (1992), pp. 139-60.

51 Arrhenius, 'Future Generations', pp. 97-100.

${ }^{52}$ Cf. Parfit, Reasons and Persons, pp. 419-42 (ch. 19). 
where $\boldsymbol{a}$ is weakly superior to $\boldsymbol{y}$. Hence, the view cannot comply with The Inequality Aversion Condition.

The Inequality Aversion Condition is widely assumed to be very plausible. ${ }^{53}$ However, if one shares the basic ranking that a certain number of people, $n$, at a certain high level, $\boldsymbol{a}$, is better than a very much larger number $(n+m)$ at a level $\boldsymbol{y}$ where life is just worth living, it might not be unreasonable to deny The Inequality Aversion Condition. It should be accepted that a population combined of $n \boldsymbol{a}$ and $m \boldsymbol{z}$, where $\boldsymbol{z}$ is slightly worse than $\boldsymbol{y}$, is better than $n \boldsymbol{a}$ alone. But it might be claimed that, in spite of the equality obtained by the move from $n \boldsymbol{a}$ combined with $m \boldsymbol{z}$ to $(n+m) \boldsymbol{y}$, no number of people gaining slightly more than $\boldsymbol{z}$ can compensate the great loss of the $n$ people. Therefore, I am not inclined to attach great weight to Arrhenius's objection.

\section{A DIFFERENT SUGGESTION}

Let me briefly mention a suggestion, which I believe Jonathan Glover was the first to make: ${ }^{54}$

But the concession that, other things being equal, there is value in extra happy people need not commit us to a simple policy of maximizing happiness. [...] It is open to us to say that one thing we value is total happiness [...] without simply adopting the total view. For we may decide that we value people's lives having various qualities (which would put them high on the scale of 'worthwhile life') and that the absence of these qualities cannot be compensated for by any numbers of extra worth-while lives without them. [...]

So we can think that extra people with lives worth living are in themselves a good thing, without having to allow that there is always some number of people whose existence outweighs any particular impoverishment of life.

Parfit has made a similar suggestion. ${ }^{55}$

Consider what I shall call the best things in life. These are the best kinds of creative activity and aesthetic experience, the best relationships between different people, and the other things which do most to make life worth living [...]

Why is it so hard to believe that my imagined world Z [...] would be better than a world of ten billion people, all of whom have an extremely high quality of life? This is hard to believe because in $\mathrm{Z}$ two things are true: people's lives are barely worth living, and most of the good things in life are lost. [...]

What we might appeal to is [...] Perfectionism. [...] We might claim that, even if some change brings a great net benefit to those who are affected, it is a change for the worse if it involves the loss of one of the best things in life.

${ }^{53}$ In fact, it is a weak statement of the requirement I mentioned in n. 3.

54 Jonathan Glover, Causing Death and Saving Lives (Harmondsworth, 1977), pp. $70-1$.

${ }_{55}$ Derek Parfit, 'Overpopulation and the Quality of Life', Applied Ethics, ed. P. Singer (Oxford, 1986), pp. 161-3. 
The idea seems to be that, apart from the total of welfare, there is a separate consideration (Parfit calls it Perfectionism) concerning the various qualities in people's lives. And there is a superiority relation between them, such that a sufficiently high level of these qualities cannot be compensated by the existence of extra people without these qualities, whatever their number.

Whereas in the previous section the evaluation was based on a basic ranking of outcomes, it is here based on two considerations that have to be weighed up against each other. Since the total of welfare approaches infinity when the number of people increases, the level of sufficiently high quality will have to be infinitely better than the level of insufficiently high quality just below. Such a dramatic discontinuity might not be plausible. Apart from that, this view will have the same implication as the Lexical View which I mentioned in the Introduction: it will put less weight on people at a low level, and this might be hard to justify.

\section{CONCLUSION}

I have demonstrated that weak superiority behaves very differently, depending on whether value is additive or not. In an additive context, weak superiority collapses into superiority, which in this context is a radical difference. In a non-additive context, the inferior value has diminishing marginal value converging to zero, such that when the value increases, it approaches a finite upper bound. In the latter context, weak superiority is not a radical difference in value; and even superiority is not a radical difference in value if it is in-between the superior and the inferior object, some element is weakly superior to another one.

Often, this difference is overlooked. For instance, Griffin appears on the one hand to appeal to the larger plausibility of weak superiority between values and at the same to hint at infinite value differences. But it is not possible to have both. Also, I have demonstrated that in moving to an additive context, rather strong additional assumptions are needed. Finally, I have also demonstrated the consequences of using weak superiority to block the Repugnant Conclusion in an additive, a non-additive and a mixed context respectively. In the first case (where weak superiority collapses), the Repugnant Conclusion is, strictly speaking, not avoided. It is just that below any finite level, there are lives definitely worse. In the second case, the implication is that adding more people at low levels has diminishing marginal value converging to zero. In the third case, there is a dramatic discontinuity in the consideration concerning the various qualities of people's lives. 
None of these implications is plausible. However, since it is hard to come up with coherent theories in the field of population ethics, weak superiority perhaps retains some interest. ${ }^{56}$

kkje@life.ku.dk

56 Parts of this article were presented at the John Stuart Mill Bicentennial Conference in April 2006, London. I am indebted to Statens Räddningsverk for financial support. I should like to thank Gustaf Arrhenius, John Broome, Roger Crisp, James Griffin, Nils Holtug and Wlodek Rabinowicz for helpful discussion on this subject, which I have worked with on and off for many years. A very early version appeared in my $\mathrm{PhD}$ thesis. A rather recent version of the article appeared in the electronic Festschrift for Wlodek Rabinowicz on his sixtieth birthday.

\section{CAMBridge JUURNALS}

